\author{
Julien Textoris \\ Thierry Calandra \\ Frédéric Pène
}

\section{A revival for immunoglobulin therapy in septic shock?}

Received: 15 September 2014

Accepted: 23 September 2014

Published online: 30 October 2014

(C) Springer-Verlag Berlin Heidelberg and ESICM 2014

\section{J. Textoris}

Intensive Care Unit, Hôpital Edouard Herriot, Hospices Civils de Lyon, Lyon, France

\section{J. Textoris $(\square)$}

Joint Research Unit bioMérieux/HCL, Pavillon P, Hôpital Edouard Herriot, BioMérieux, 5 place d'Arsonval, Marcy l'Etoile, 69437 Lyon Cedex 03, France

e-mail: julien.textoris@gmail.com

Tel.: +33472119546

T. Calandra

Infectious Diseases Service, Department of Medicine, Centre

Hospitalier Universitaire Vaudois, Lausanne, Switzerland

T. Calandra

University of Lausanne, Lausanne, Switzerland

F. Pène

Medical ICU, Cochin hospital, Assistance Publique-Hôpitaux de Paris, Paris, France

F. Pène

University Paris Descartes, Paris, France

As of 13 September 2014, World Sepsis Day, sepsis remains a leading cause of death worldwide. Over the last decade, the implementation of guidelines or bundles has had a major impact on the management of sepsis patients, stressing the need for prompt diagnosis and therapy including (1) early administration of antibiotics and source control, (2) hemodynamic resuscitation, and (3) prevention and management of organ failures. Besides antimicrobial therapy and supportive care, no immunomodulatory strategy has yielded clear-cut and reproducible results in terms of improved survival [1].

Polyvalent intravenous immunoglobulin (IVIG) preparations, that contain primarily IgGs, have long been used in various clinical situations. For example, IVIG has been shown to be efficacious when used as a replacement therapy in primary or secondary humoral immunodeficiencies or as an immunomodulatory therapy in a number of autoimmune and inflammatory diseases such as primary immune thrombocytopenia, Guillain-Barré syndrome, and Kawasaki's disease including severe clinical presentations with multiple organ dysfunction syndrome [2]. In addition, IVIG has also been used as an adjuvant treatment of sepsis given its potential effects both on pathogens and the host's response. How IVIG therapy may modulate the immune system in autoimmune diseases and in sepsis has been nicely reviewed elsewhere $[3,4]$. Potential mechanisms of action of IVIG therapy in sepsis include (1) a role in pathogen recognition, clearance, and toxin scavenging; (2) modulation of pattern recognition receptors (inflammasomes), signalling pathways (NF- $\kappa \mathrm{B}$ ), and effector molecules (cytokines) of the immune response; and (3) a direct anti-apoptotic effect on immune cells.

IgM-enriched IVIG preparation exhibit additional immunomodulatory properties when compared with standard IVIG. Soluble IgM consists primarily of pentamers and are divided into natural and immune IgM. Natural IgM antibodies are constitutively secreted by B1 cells. Several elegant animal models have demonstrated the protective role of natural IgM against numerous viral, bacterial, and parasitic infections (Influenza, Pseudomonas aeruginosa, Plasmodium falciparum) and a worse outcome of patients with IgM deficiency [5-7]. IgM has an important role in the classical pathway of complement activation when complexed with antigens and its 
pentameric structure favors agglutination of the invading organism and its neutralization. IgM also favors interactions between pathogens and phagocytic cells, resulting in enhanced innate immune activation and improved antigen presentation. Recent reports have also identified a potential role of $\operatorname{IgM}$ in the maintenance of tissue homeostasis, through a C1q-mediated clearance of apoptotic and altered cells, and inhibition of inflammation (Fig. 1) [8,9].

Unfortunately the therapeutic potential of IVIG did not clearly translate into improved outcomes in septic patients. A number of prospective studies that addressed the efficacy of polyclonal IVIG in the treatment of septic shock have been included in several meta-analysis, including the recently updated one from the Cochrane Collaboration [10]. A primary analysis indicated a benefit of IVIG in survival that seemed even more pronounced when using IgM-enriched preparations. However, the interpretation of meta-analysis relies on several factors including an extensive assessment of the risk of bias, the respective weight related to the number of patients, as well as the marked influence of single-center studies on outcomes [11, 12]. Restriction to high-quality studies with a low risk of confounding factors did not show any benefit from IVIG administration with both standard and IgMenriched preparations [10]. Most importantly, the heterogeneous quality of IVIG preparations may represent a major but non-measurable bias across studies [13]. The main conclusion from the meta-analysis was that the current evidence for benefits was inconsistent and inconclusive, and indeed the latest version of the Surviving Sepsis Campaign guidelines in 2012 stated: "we suggest not using intravenous immunoglobulins in adult patients with severe sepsis or septic shock (grade 2B)" [14].
An article recently published in Intensive Care Medicine is reviving the interest in IVIG for the treatment of septic shock. In a single-center retrospective study, Cavazzuti and colleagues [15] analyzed a cohort of septic shock patients who did (92 patients) or did not (76 patients) receive early treatment with polyclonal IgMenriched IVIG in addition to common 6-h and 24-h bundles. Routine administration of IgM-enriched IVIG preparations in septic patients was based on a published meta-analysis suggesting a survival benefit [10]. Administration of $\operatorname{IgM}$ was associated with a dramatic and impressive $21.1 \%$ absolute improvement in 30-day survival (75 vs. $53.9 \%$ ) that persisted after appropriate adjustment through multivariate analysis as well as a propensity score-matching. Although the authors performed the most accurate and robust analysis of the retrospective data, their findings must be read with caution. The main limits of the study lie in its retrospective design and in the core question of the decision-making process of treatment allocation. Comparison between IVIG-treated and non-treated patients did not identify any significant baseline differences. However, some uncollected baseline characteristics, such as functional and nutritional status, as well as specific prognostic factors of underlying comorbidities may have influenced the decision whether or not to administer IVIG therapy. The study was not designed to address the mechanism of action of IgM-enriched preparations, but a decrease in the incidence of refractory shock and multiple organ failure in IgM-treated patients would grossly argue in favor of an anti-inflammatory role of IgM-enriched preparation. One can regret the lack of baseline measurement of total Ig levels as well as the distribution of Ig subsets prior to exogenous IVIG administration. Indeed, it has been reported that transient hypogammaglobulinemia may
Fig. 1 Potential mechanisms of action of IgM-enriched IVIG in sepsis. 1 Scavenging effect on toxins or inflammatory mediators, 2 pathogen recognition and clearance through enhanced complement activation and phagocytosis, 3 direct anti-apoptotic effect on lymphocytes, 4 IgM-mediated clearance of apoptotic cells. The question mark denotes unclear interactions still under evaluation

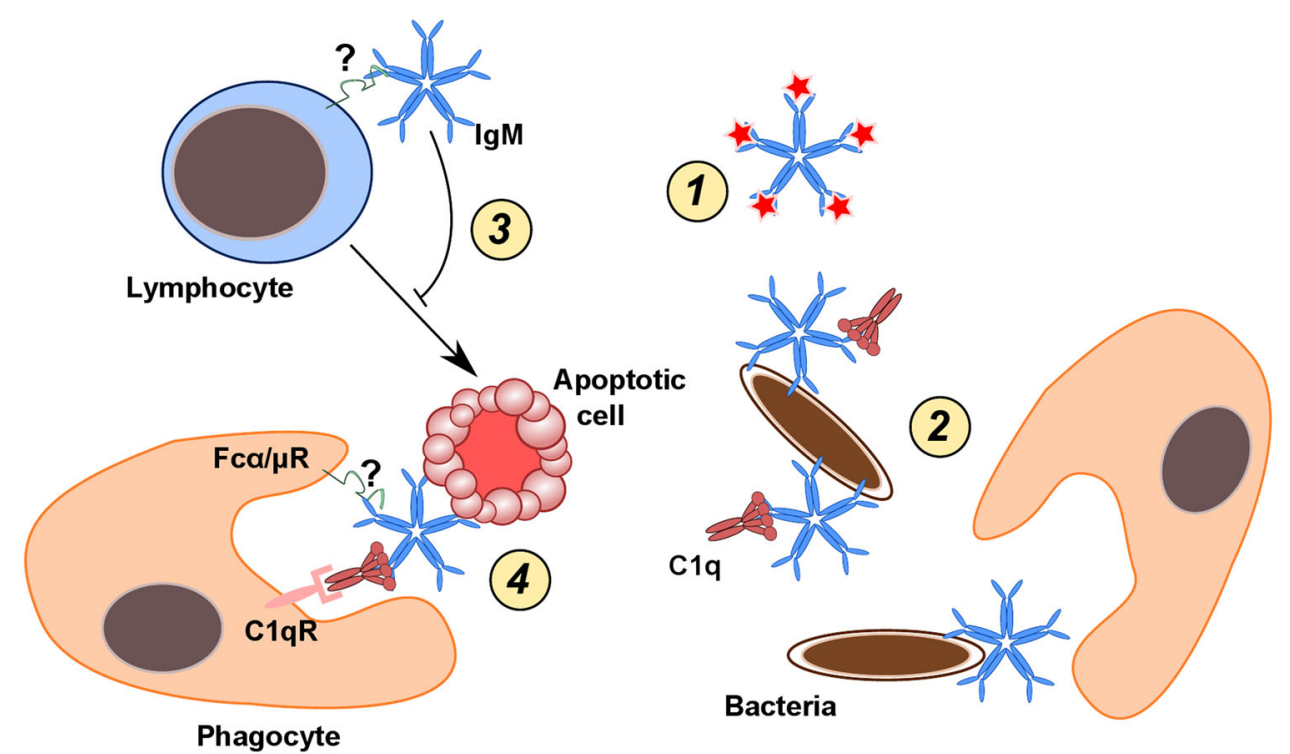


arise within the first days of septic shock and may be associated with increased mortality [16].

Albeit interesting, the results of this retrospective single-center study are unlikely to challenge the results observed in prospective randomized clinical trials and to modify the current evidence against the use of IVIG in sepsis. But one must admit that the dramatic survival differences between treated and non-treated patients is intriguing enough to suggest a specific therapeutic effect of IgM-enriched IVIG preparations and to support further prospective interventional studies. Spin-off studies aimed at assessing the immune mechanisms whereby IgM-enriched IVIG preparations exert their effects would be welcome. This translational approach is essential for a careful evaluation of biological plausibility and mode of action of immunomodulatory therapies of sepsis, and may also help to identify which patients are likely to benefit from them.

Conflicts of interest None.

\section{References}

1. Hotchkiss RS, Monneret G, Payen D (2013) Sepsis-induced immunosuppression: from cellular dysfunctions to immunotherapy. Nat Rev Immunol 13:862-874

2. Gatterre P, Oualha M, Dupic L, Iserin F, Bodemer C, Lesage F, Hubert P (2012) Kawasaki disease: an unexpected etiology of shock and multiple organ dysfunction syndrome. Intensive Care Med 38:872-878

3. Schwab I, Nimmerjahn F (2013) Intravenous immunoglobulin therapy: how does IgG modulate the immune system? Nat Rev Immunol 13:176-189

4. Shankar-Hari M, Spencer J, Sewell WA, Rowan KM, Singer M (2012) Bench-to-bedside review: immunoglobulin therapy for sepsisbiological plausibility from a critical care perspective. Crit Care 16:206

5. Jayasekera JP, Moseman EA, Carroll MC (2007) Natural antibody and complement mediate neutralization of influenza virus in the absence of prior immunity. J Virol 81:3487-3494

6. Kinoshita M, Shinomiya N, Ono S, Tsujimoto H, Kawabata T, Matsumoto A, Hiraide H, Seki S (2006) Restoration of natural IgM production from liver B cells by exogenous IL-18 improves the survival of burn-injured mice infected with Pseudomonas aeruginosa. J Immunol 177:4627-4635

7. Ghumra A, Semblat JP, McIntosh RS, Raza A, Rasmussen IB, Braathen R, Johansen FE, Sandlie I, Mongini PK, Rowe JA, Pleass RJ (2008) Identification of residues in the $\mathrm{Cmu} 4$ domain of polymeric IgM essential for interaction with Plasmodium falciparum erythrocyte membrane protein 1 (PfEMP1). J Immunol 181:1988-2000
8. Quartier P, Potter PK, Ehrenstein MR, Walport MJ, Botto M (2005) Predominant role of IgM-dependent activation of the classical pathway in the clearance of dying cells by murine bone marrow-derived macrophages in vitro. Eur J Immunol 35:252-260

9. Notley CA, Brown MA, Wright GP, Ehrenstein MR (2011) Natural IgM is required for suppression of inflammatory arthritis by apoptotic cells. J Immunol 186:4967-4972

10. Alejandria MM, Lansang MA, Dans LF, Mantaring JB 3rd (2013) Intravenous immunoglobulin for treating sepsis, severe sepsis and septic shock. Cochrane Database Syst Rev 9:CD001090

11. Dechartres A, Boutron I, Trinquart L, Charles P, Ravaud P (2011) Singlecenter trials show larger treatment effects than multicenter trials: evidence from a meta-epidemiologic study. Ann Intern Med 155:39-51

12. Dechartres A, Altman DG, Trinquart L, Boutron I, Ravaud P (2014) Association between analytic strategy and estimates of treatment outcomes in metaanalyses. JAMA 312:623-630

13. Gelfand EW (2006) Differences between IGIV products: impact on clinical outcome. Int Immunopharmacol 6:592-599

14. Dellinger RP, Levy MM, Rhodes A, Annane D, Gerlach H, Opal SM, Sevransky JE, Sprung CL, Douglas IS, Jaeschke R, Osborn TM, Nunnally ME, Townsend SR, Reinhart K, Kleinpell RM, Angus DC, Deutschman CS, Machado FR, Rubenfeld GD, Webb S, Beale RJ, Vincent JL, Moreno R (2013) Surviving Sepsis Campaign: international guidelines for management of severe sepsis and septic shock, 2012. Intensive Care Med 39:165-228
15. Cavazzuti I, Serafini G, Busani S, Rinaldi L, Biagioni E, Buoncristiano M, Girardis M (2014) Early therapy with IgM-enriched polyclonal immunoglobulin in patients with septic shock. Intensive Care Med. doi: 10.1007/s00134-014-3474-6

16. Venet F, Gebeile R, Bancel J, Guignant C, Poitevin-Later F, Malcus C, Lepape A, Monneret G (2011) Assessment of plasmatic immunoglobulin $\mathrm{G}, \mathrm{A}$ and $\mathrm{M}$ levels in septic shock patients. Int Immunopharmacol 11:2086-2090 